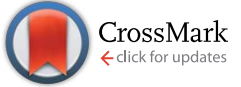

Cite this: RSC Adv., 2017, 7, 1396

Received 4th November 2016 Accepted 25th November 2016

DOI: 10.1039/c6ra26303h

www.rsc.org/advances

\section{Fluorometric "Turn-On" glucose sensing through the in situ generation of silver nanoclusters $\uparrow$}

\author{
Yang Chen, ${ }^{* a}$ Yuanqing Sun, ${ }^{\text {ab }}$ Rongjun Song, ${ }^{a}$ Shanliang Song, ${ }^{b}$ Yue Zhao, ${ }^{b}$ \\ Xudong Yang, ${ }^{\mathrm{b}}$ Cong Y $\mathrm{u}^{\mathrm{c}}$ and Quan Lin ${ }^{* \mathrm{~b}}$
}

Fluorometric "turn-on" glucose detection is presented that is based on the Fenton reaction which can trigger the generation of $\mathrm{Ag}$ nanoclusters (Ag NCs). Oxidation of $\mathrm{D}$-glucose by glucose oxidase generates $\mathrm{H}_{2} \mathrm{O}_{2}$. Reactive radicals are formed from the Fenton reaction of $\mathrm{H}_{2} \mathrm{O}_{2}$ and $\mathrm{Fe}^{2+}$. These radicals initiate the polymerization of methacrylic acid to form poly(methacrylic acid) which is used as a template to generate fluorescent Ag NCs in situ by illumination with UV light. The increase in Ag NCs fluorescence intensity can be used to quantify glucose concentration. The sensing approach employed here provides a new strategy for the determination of glucose selectively.

\section{Introduction}

Fluorescent metal nanoclusters (NCs), especially those made of gold and silver, are a class of nanomaterials consisting of only several to tens of atoms. The size of the NCs is typically $\sim 1 \mathrm{~nm}$, which is comparable to the Fermi wavelength of electrons. ${ }^{1 a}$ The strong quantum confinement of free electrons show obvious effects on these ultra-small particles, and new physical and chemical properties emerge. ${ }^{1 b}$ Owing to their ultrasmall size, the NCs give rise to discrete electronic energy levels and unique geometric structures, which are significantly different from the larger nanoparticles (NPs). ${ }^{1}$ As a result, metal NCs exhibit unique molecular-like properties, such as welldefined molecular structures and strong fluorescence. ${ }^{1}$ These NCs have received great attentions in recent years due to their unique optical, electrical, and chemical properties. They exhibit excellent features such as strong photoluminescence, size-dependent tunable emission, high photoluminescence quantum yield, excellent photo-stability, and good biocompatibility. ${ }^{2}$ The emissive NCs offer a new platform for the construction of various novel biosensors. ${ }^{3}$

Silver nanoclusters are generally fluorescent upon photoexcitation. They have been widely used in many fields, such as biosensing, bioimaging, antibacterial agent, and cancer radiotherapy. ${ }^{4,5}$ Template-based synthesis is an efficient and facile

${ }^{a}$ College of Science, Northeast Forestry University, Harbin, 150040, P. R. China. E-mail:ychen@nefu.edu.cn

${ }^{b}$ State Key Laboratory of Supramolecular Structure and Materials, College of Chemistry, Jilin University, Changchun, 130012, P. R. China. E-mail: linquan@jlu. edu.cn

${ }^{c}$ State Key Laboratory of Electroanalytical Chemistry, Changchun Institute of Applied Chemistry, Chinese Academy of Sciences, Changchun, 130022, P. R. China

$\uparrow$ Electronic supplementary information (ESI) available: Experimental details and supplementary figures. See DOI: 10.1039/c6ra26303h method to prepare highly fluorescent Ag NCs. The frequently used templates are nucleic acids, polymers, peptides, proteins, and free thiol containing molecules. ${ }^{6}$ Common routes for the synthesis of water dispersible fluorescent Ag NCs employ the reduction of silver salt precursors containing a suitable template through a chemical, photochemical, or sonochemical reduction procedure. ${ }^{7}$

Glucose is an important carbohydrate in biology. It is a basic necessity of human and plays vital roles in body metabolism. Cells use it as energy source and a metabolic intermediate in most organisms. The concentration of glucose is maintained within a proper range through the interplay of several endocrine and neural glucostatic systems. ${ }^{8}$ An abnormal level of glucose can cause severe diseases such as diabetes. Therefore, glucose detection is of great importance in life science and clinical analysis. ${ }^{9}$ And the construction of an efficient glucose sensor is of great significance.

A variety of methods have been proposed for the accurate estimation of glucose concentration, such as the electrochemical, chemiluminescent, colorimetric, surface-enhanced Raman scattering, and fluorometric assays. ${ }^{10}$ Among these methods, fluorometric methods have been well established. ${ }^{\mathbf{1 0 e}, \mathbf{1 1}}$ Recently, selective detection of glucose level using fluorescent metal nanoclusters has drawn considerable attentions. ${ }^{\mathbf{4 a , 1 2}}$ However, most of the methods employed existing NCs. In addition, a signal-off detection mode was used. The fluorescence of the NCs was usually quenched by $\mathrm{H}_{2} \mathrm{O}_{2}$ that generated from the enzymatic oxidation of glucose, which could considerably increase the likelihood of false positive signals associated with the signal-off detection.

Herein, we report that for the first time, a fluorometric "turnon" glucose assay is constructed based on the in situ generation of $\mathrm{Ag}$ NCs which use the Fenton reaction product poly(methacrylic acid) (PMAA) as a template. The increase in 
emission intensity of the NCs solution provides a facile way for the sensing of glucose. Our method exhibits several remarkable characteristics: (1) highly fluorescent Ag NCs were generated in situ; (2) avoided the use of organic functional molecules, which considerably simplified the sensing procedures; (3) avoided the introduction of boric acid functionalities to the nanomaterials; (4) a fluorometric "turn-on" detection of glucose was constructed. This fluorescent nanocluster-based label-free method provides a new platform for selective and cost-effective detection of glucose.

\section{Experimental}

\section{Materials}

Methacrylic acid (MAA) was purchased from J\&K Chemical Technology (Beijing, China). D-Glucose monohydrate, glucose oxidase (GOx), dialysis membrane (1000 MWCO) were purchased from Sangon Biotech Co., Ltd. (Shanghai, China). Ferrous sulfate heptahydrate $\left(\mathrm{FeSO}_{4} \cdot 7 \mathrm{H}_{2} \mathrm{O}\right)$ was purchased from Beijing Chemical Works (Beijing, China). Silver nitrate $\left(\mathrm{AgNO}_{3}\right)$ was purchased from Sinpharm Chemical Reagent Co., Ltd. (Beijing, China). The ultra-pure water used throughout the experiments was purified with a Milli-Q A10 filtration system (Millipore, Billerica, MA, USA). MAA was purified through vacuum distillation at $60{ }^{\circ} \mathrm{C}$ and stored in a refrigerator $\left(-20^{\circ} \mathrm{C}\right)$ before use. All other reagents were of analytical grade and used without further purification.

\section{Characterization}

Emission spectra were recorded on a Fluoromax-4 spectrofluorometer (Horiba Jobin Yvon Inc., USA). Excitation wavelength was $480 \mathrm{~nm}$ and the emission spectra were corrected against photomultiplier tube (PMT) response. Excitation and emission slit widths were both of $5 \mathrm{~nm}$. UV-Vis absorption spectra were collected using a Cary 50 Bio Spectrophotometer (Varian Inc., CA, USA). Quartz cuvettes with $10 \mathrm{~mm}$ path length and $2 \mathrm{~mm}$ window width were used for fluorescence and UV-Vis measurements. X-ray photoelectron spectroscopy (XPS) spectra were obtained using a VG Thermo ESCALAB 250 spectrometer (VG Scientific) operated at $120 \mathrm{~W}$. Transmission electron microscopy (TEM) measurements were performed on a FEI TECNAI G2 high-resolution transmission electron microscope (the Netherlands) operated at an accelerating voltage of $200 \mathrm{kV}$. Samples were prepared by placing a drop of the Ag NCs solution onto a carbon-coated copper grid and then dried at room temperature. UV light source was from the UVP GelDoc-It TS Imaging System (Upland, CA, USA). All spectra were taken at an ambient temperature of $22{ }^{\circ} \mathrm{C}$ unless otherwise specified.

\section{Fenton reaction triggered generation of Ag NCs for glucose sensing}

A typical experimental procedure is as follows: different volumes of D-glucose were mixed with $20 \mu \mathrm{L} \mathrm{GOx}\left(0.1 \mathrm{mg} \mathrm{mL}^{-1}\right)$, $20 \mu \mathrm{L}$ citrate buffer $(100 \mathrm{mM}, \mathrm{pH} 5.5)$, and a certain amount of water. The samples were incubated at $37{ }^{\circ} \mathrm{C}$ for $2 \mathrm{~h}$. Final concentrations: $5 \mu \mathrm{g} \mathrm{mL} \mathrm{mL}^{-1}$ GOx. Final sample volume: $400 \mu \mathrm{L}$.
$40 \mu \mathrm{L}$ MAA $(5 \mathrm{M}), 10 \mu \mathrm{L} \mathrm{FeSO}_{4}(50 \mathrm{mM})$, and $50 \mu \mathrm{L}$ citrate buffer (100 mM, pH 3.0) were added to the $400 \mu \mathrm{L}$ sample mixtures. The sample solutions were left standing for $30 \mathrm{~min}$. Final concentrations: $400 \mathrm{mM}$ MAA, $1 \mathrm{mM} \mathrm{FeSO}_{4}$. Final sample volume: $500 \mu \mathrm{L}$. The solutions were then dialyzed against water overnight.

$200 \mu \mathrm{L}$ of the aforementioned polymerization solution, $80 \mu \mathrm{L}$ $\mathrm{AgNO}_{3}(100 \mathrm{mM}), 40 \mu \mathrm{L} \mathrm{HAc}-\mathrm{NaAc}$ buffer (100 mM, pH 4.5), and $80 \mu \mathrm{L}$ water were mixed together. The solutions were then irradiated under UV light $(302 \mathrm{~nm}, 8 \mathrm{~W} \times 4)$ for $4 \mathrm{~min}$. Fluorescence spectra were measured immediately after the irradiation. Final sample volume: $400 \mu \mathrm{L}$.

All the experiments were repeated three times.

\section{Results and discussion}

The detection strategy is schematically depicted in Scheme 1. The enzymatic oxidation of D-glucose in the presence of $\mathrm{O}_{2}$ could generate $\mathrm{H}_{2} \mathrm{O}_{2}$, which can oxidize $\mathrm{Fe}^{2+}$ in the Fenton reagent. The Fenton reaction leads to the generation of radicals, which initiate the polymerization of MAA. The polymerization product PMAA can be used as a template to prepare the fluorescent Ag NCs under UV illumination. The oxidation of glucose leads to these series of reactions and fluorescent Ag NCs are generated as a result. The changes in emission intensity of the $\mathrm{Ag}$ NCs are directly related to glucose concentration.

Literature reports have showed that the enzymatic oxidation of glucose can generate $\mathrm{H}_{2} \mathrm{O}_{2},{ }^{4 a, 9}$ which can in turn generate reactive radicals. ${ }^{\mathbf{1 0 c}, \mathbf{1 3}}$ In order to demonstrate that the fluorescent Ag NCs can only be generated in the presence of the polymerization product PMAA under our experimental conditions, control experiments were carried out. The results show that a commercial PMAA $\left(M_{\mathrm{w}}=6500\right)$ can be used as a template to prepare the fluorescent Ag NCs (Fig. S1, ESI $\dagger$ ). Fig. S2 $\uparrow$ shows that no fluorescent $\mathrm{Ag}$ NCs formation was observed in the absence of glucose. When GOx, MAA and $\mathrm{Ag}^{+}$were mixed, hardly any NCs fluorescence was observed. This is because that no $\mathrm{H}_{2} \mathrm{O}_{2}$ was generated, which could not induce the Fenton reaction, and no PMAA formed as a result. Similar result was obtained in the absence of GOx. Glucose and GOx alone also cannot induce the formation of the fluorescent Ag NCs. Sample

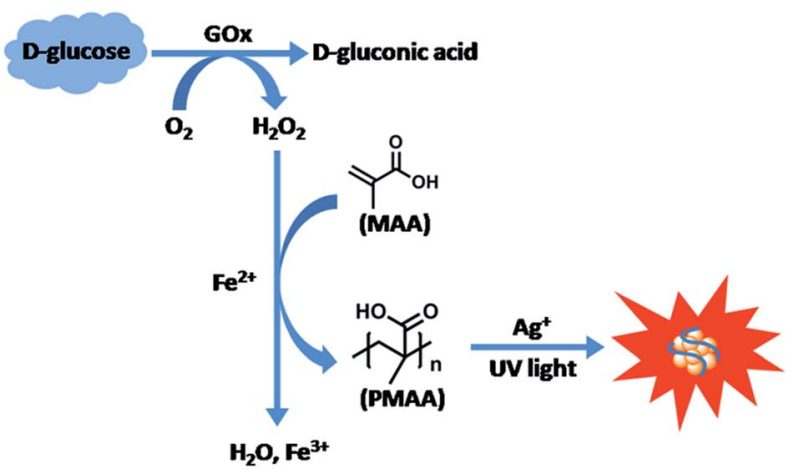

Scheme 1 Schematic illustration of the Fenton reaction triggered generation of fluorescent Ag NCs for glucose sensing. 
4 shows that no NCs emission was observed without the addition of the MAA monomer. Similar result was observed in sample 5. It indicates that MAA itself cannot induce the formation of the Ag NCs. The fluorescent Ag NCs were prepared when only the MAA polymerization was initiated through the Fenton reaction.

Fluorescent silver nanoclusters were prepared through the photochemical reduction procedure. The brown solution of the Ag NCs emits intense orange fluorescence under $365 \mathrm{~nm}$ UV light (Fig. 1, inset photos). The fluorescent Ag NCs show an emission with peak maximum at $585 \mathrm{~nm}$ under $480 \mathrm{~nm}$ excitation (Fig. 1). The size distribution of the resultant fluorescent $\mathrm{Ag}$ NCs was characterized by TEM. As shown in Fig. 2, the average size of the NCs is $1.64 \mathrm{~nm}$. No large Ag particles or aggregates were detected by TEM. The absorption band of the $\mathrm{Ag}$ NCs is located at $510 \mathrm{~nm}$ (Fig. S3†). The absence of large $\mathrm{Ag}$ nanoparticles led to pure NCs absorption spectrum without surface plasmon bands in the $400-450 \mathrm{~nm}$ region. ${ }^{14} \mathrm{X}$-ray photoelectron spectroscopy (XPS) study was carried out to analyze the valence states of $\mathrm{Ag}$ (Fig. 3). The binding energy values of $\mathrm{Ag}\left(3 \mathrm{~d}_{5 / 2}\right)$ and $\mathrm{Ag}\left(3 \mathrm{~d}_{3 / 2}\right)$ are $368.3 \mathrm{eV}$ and $374.3 \mathrm{eV}$, respectively, which indicate that $\mathrm{Ag}(\mathrm{I})$ was reduced to $\mathrm{Ag}(0)$ after UV illumination. ${ }^{15}$

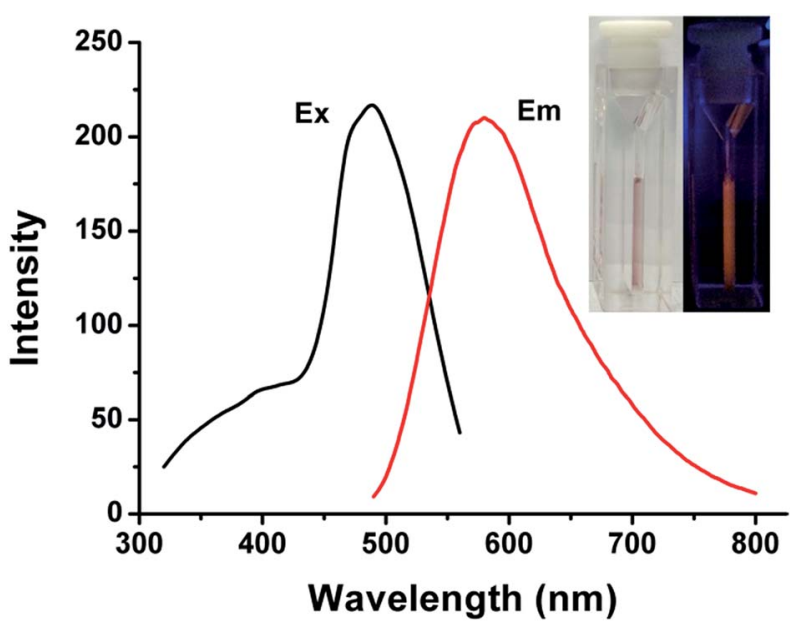

Fig. 1 Excitation and emission spectra of the Ag NCs ( $\lambda_{\mathrm{ex}}=480 \mathrm{~nm}$, $\lambda_{\mathrm{em}}=585 \mathrm{~nm}$ ). Inset: the photographs of the Ag NCs samples under visible light (left) and $365 \mathrm{~nm}$ UV light illumination (right). Conditions: $600 \mu \mathrm{M}$ glucose, 400 mM MAA, 4 min irradiation time.
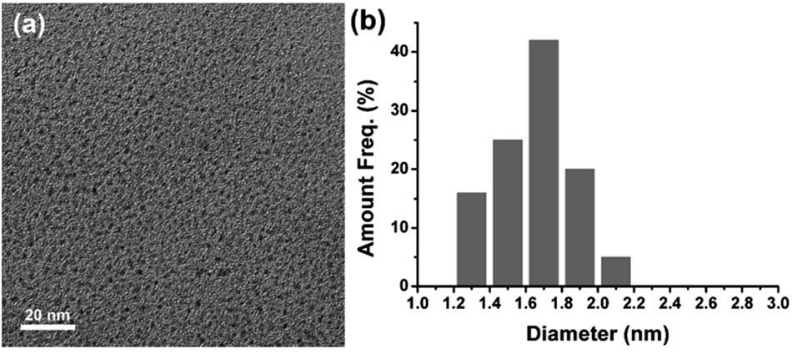

Fig. 2 (a) TEM image of the Ag NCs, and (b) the corresponding size distribution of the Ag NCs. Conditions: $600 \mu \mathrm{M}$ glucose, $400 \mathrm{mM} \mathrm{MAA}$, 4 min irradiation time.

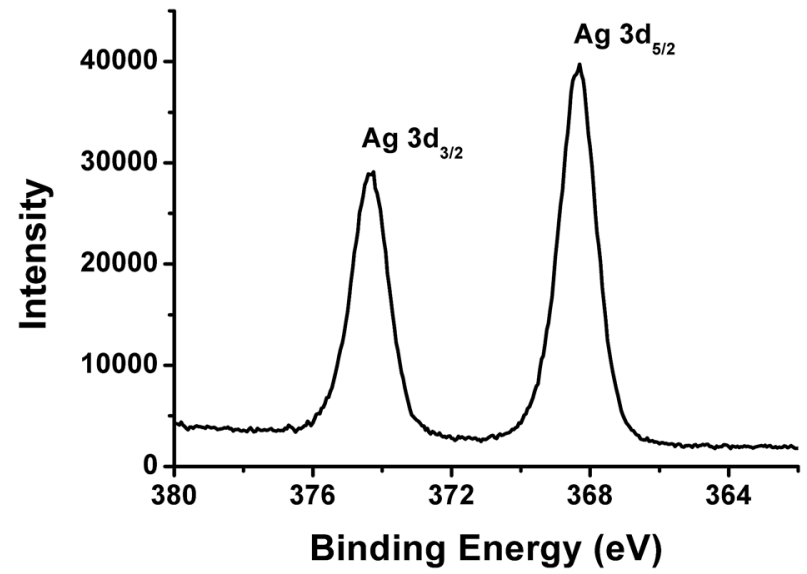

Fig. 3 XPS spectrum of the Ag NCs. Conditions: $600 \mu \mathrm{M}$ glucose, 400 mM MAA, 4 min irradiation time.

We have investigated the optimum assay conditions such as the MAA concentration and the illumination time in order to get the best sensing performance. MAA of various concentrations (0-500 $\mathrm{mM})$ were tested, and the possible formation of the $\mathrm{Ag}$ NCs was estimated by measurement of the changes in
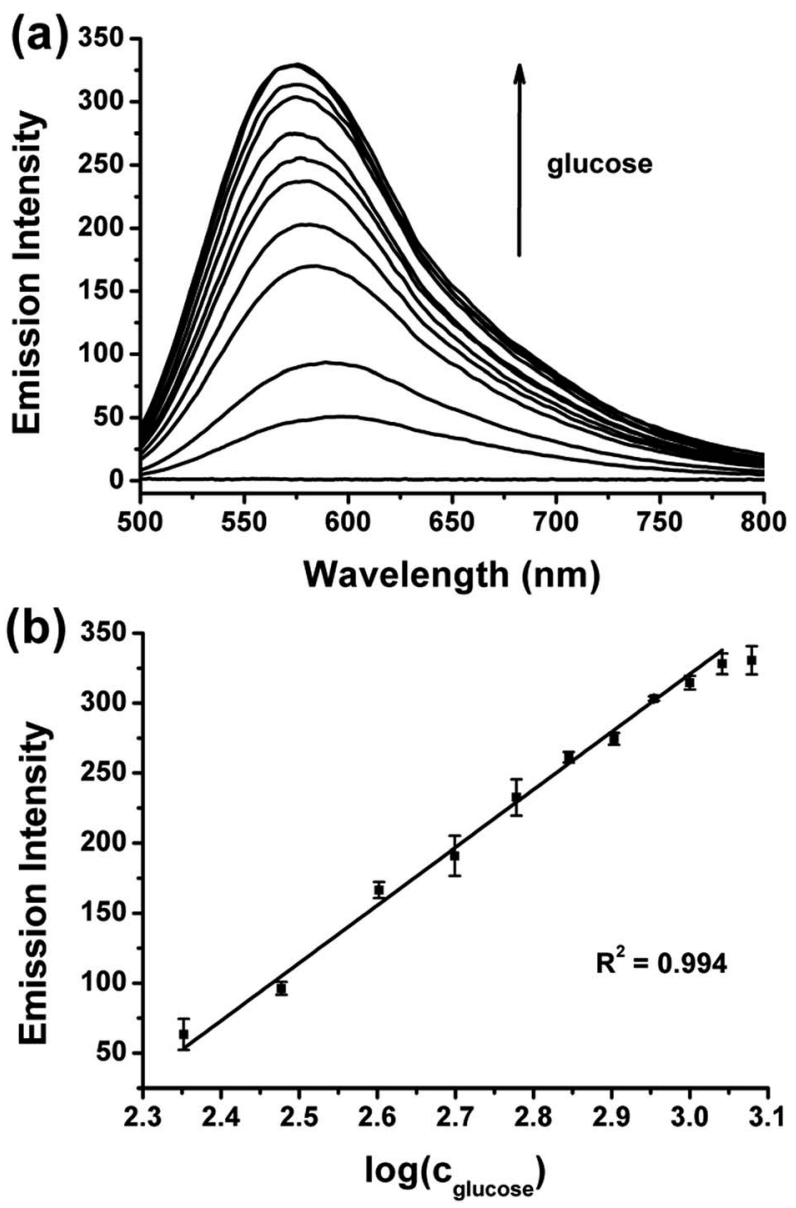

Fig. 4 (a) Changes in emission spectrum as a function of glucose concentration ( $\lambda_{\mathrm{ex}}=480 \mathrm{~nm}$ ), (b) changes in maximum emission intensity of (a) versus glucose concentration. 


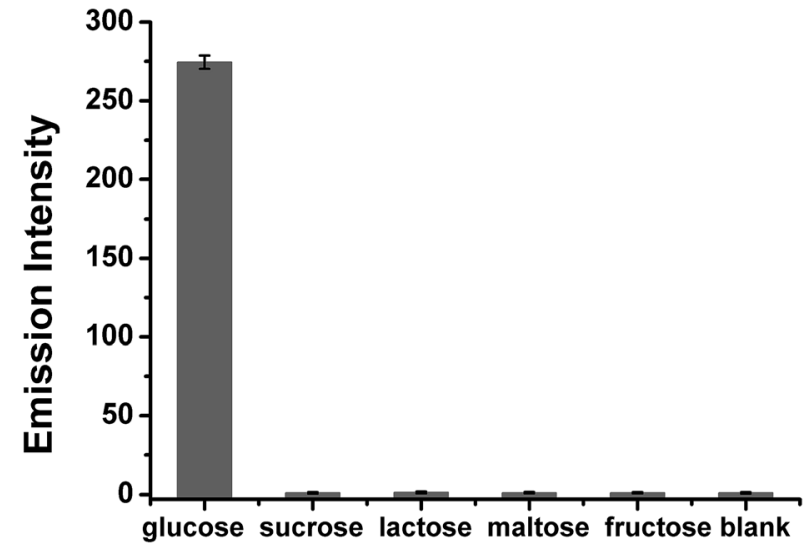

Fig. 5 Selectivity of the assay. Concentrations of glucose and the other sugars: $600 \mu \mathrm{M}$ each.

emission spectra (Fig. S4†). The results show that at lower concentrations of MAA $(\leq 100 \mathrm{mM})$, very weak Ag NCs emission was observed. With the increase of MAA concentration, the NCs emission increased, and the emission intensity reached a maximum value when $400-500 \mathrm{mM}$ of MAA was used. The optimized MAA concentration of $400 \mathrm{mM}$ was therefore used at current investigation.

The changes in emission spectra of the $\mathrm{Ag}$ NCs with UV illumination time are displayed in Fig. S5. $\dagger$ Prior to UV illumination, the fluorescence spectrum shows no obvious emission. With the increase of the illumination time, increased emission intensity of the $\mathrm{Ag}$ NCs was observed. Strongest emission was observed at 4 minutes illumination. Prolonged UV illumination led to decreased emission of the Ag NCs, which may be a result of the formation of larger non-fluorescent Ag nanoparticles. ${ }^{7 b, 14 b, 15 a}$ Four minutes UV illumination was therefore selected for the preparation of the Ag NCs.

Glucose determination experiments were conducted under the optimized assay conditions. Fig. 4 shows that with the increase of the glucose concentration, increased Ag NCs emission was observed. The emission intensity changes of the Ag NCs were in direct proportion to the logarithm of the glucose concentration in the range of 225-1100 $\mu \mathrm{M}$. However, we face the challenge to extend our sensing system to real samples. As free radicals are not stable, they may be quenched in the complicated system, and the monomer MAA cannot be initiated as a result. To address the selectivity of the assay, four sugars (sucrose, lactose, maltose, and fructose) were tested (Fig. 5). The results show that none of them had the ability to induce the formation of the $\mathrm{Ag}$ NCs. Thus our assay is quite selective for glucose.

\section{Conclusions}

A fluorometric "turn-on" detection of glucose based on the Fenton reaction triggered generation of Ag NCs is developed. The enzymatic oxidation of glucose in the presence of $\mathrm{O}_{2}$ could generate $\mathrm{H}_{2} \mathrm{O}_{2}$. The polymerization of MAA could be initiated by the radicals that generated from the Fenton reaction. The product PMAA was used as template for the preparation of the fluorescent Ag NCs. The changes in emission intensity of the $\mathrm{Ag}$ NCs were directly related to the amount of glucose in the solution. The assay offers a new nanoscale platform for the determination of glucose. We envision that this detecting system could be used in glucose sensing related biochemical and biomedical research, which is of great significance in the early diagnosis of diseases.

\section{Acknowledgements}

This work was supported by the National Natural Science Foundation of China (51373061 and 51603018), and the Fundamental Research Funds for the Central Universities (2572015BX01).

\section{Notes and references}

1 (a) J. Zheng, P. R. Nicovich and R. M. Dickson, Annu. Rev. Phys. Chem., 2007, 58, 409; (b) K. Zheng, X. Yuan, N. Goswami, Q. Zhang and J. Xie, RSC Adv., 2014, 4, 60581. 2 (a) L. Shang, S. Dong and G. U. Nienhaus, Nano Today, 2011, 6, 401; (b) N. Goswami, Q. Yao, Z. Luo, J. Li, T. Chen and J. Xie, J. Phys. Chem. Lett., 2016, 7, 962; (c) X. Song, N. Goswami, H. Yang and J. Xie, Analyst, 2016, 141, 3126; (d) K. Zheng, X. Yuan, K. Kuah, Z. Luo, Q. Yao, Q. Zhang and J. Xie, Chem. Commun., 2015, 51, 15165.

3 (a) Y. Chen, H. Zhou, Y. Wang, W. Li, J. Chen, Q. Lin and C. Yu, Chem. Commun., 2013, 49, 9821; (b) Y. Chen, Y. Wang, C. Wang, W. Li, H. Zhou, H. Jiao, Q. Lin and C. Yu, J. Colloid Interface Sci., 2013, 396, 63; (c) Y. Chen, W. Li, Y. Wang, X. Yang, J. Chen, Y. Jiang, C. Yu and Q. Lin, J. Mater. Chem. C, 2014, 2, 4080; (d) Y. Yu, B. Y. L. Mok, X. J. Loh and Y. N. Tan, Adv. Healthcare Mater., 2016, 5, 1844.

4 (a) X. Liu, F. Wang, A. Niazov-Elkan, W. Guo and I. Willner, Nano Lett., 2013, 13, 309; (b) F. Cao, E. Ju, C. Liu, F. Pu, J. Ren and X. Qu, Chem. Commun., 2016, 52, 5167; (c) X. Ran, Z. Wang, Z. Zhang, F. Pu, J. Ren and X. Qu, Chem. Commun., 2016, 52, 557; (d) Z. Huang, Y. Tao, F. Pu, J. Ren and X. Qu, Chem.-Eur. J., 2012, 18, 6663.

5 (a) K. Zheng, M. I. Setyawati, T. P. Lim, D. T. Leong and J. Xie, ACS Nano, 2016, 10, 7934; (b) L. Zhang, Y. He, N. Goswami, J. Xie, B. Zhang and X. Tao, Chemosphere, 2016, 153, 322; (c) N. Goswami, K. Zheng and J. Xie, Nanoscale, 2014, 6, 13328; (d) Z. Luo, K. Zheng and J. Xie, Chem. Commun., 2014, 50, 5143.

6 I. Díez and R. H. A. Ras, Nanoscale, 2011, 3, 1963.

7 (a) S. Sun, H. Wang and X. Yan, Chem. Commun., 2011, 47, 3817; (b) L. Shang and S. Dong, Chem. Commun., 2008, 1088; (c) H. Xu and K. S. Suslick, ACS Nano, 2010, 4, 3209.

8 F. Rolland, J. Winderickx and J. M. Thevelein, Trends Biochem. Sci., 2001, 26, 310.

9 S. P. Nichols, A. Koh, W. L. Storm, J. H. Shin and M. H. Schoenfisch, Chem. Rev., 2013, 113, 2528.

10 (a) X. Wang, X. Dong, Y. Wen, C. Li, Q. Xiong and P. Chen, Chem. Commun., 2012, 48, 6490; (b) P. Yang, S. Jin, Q. Xu and S. Yu, Small, 2013, 9, 199; (c) C. Xu, J. Ren, L. Feng 
and X. Qu, Chem. Commun., 2012, 48, 3739; (d) K. V. Kong, Z. Lam, W. K. O. Lau, W. K. Leong and M. Olivo, J. Am. Chem. Soc., 2013, 135, 18028; (e) X. Wang, J. Hu, G. Zhang and S. Liu, J. Am. Chem. Soc., 2014, 136, 9890.

11 (a) L. Sang and H. Wang, Anal. Chem., 2014, 86, 5706; (b) P. Shen and Y. Xia, Anal. Chem., 2014, 86, 5323; (c) C. Zhang, Y. Yuan, S. Zhang, Y. Wang and Z. Liu, Angew. Chem., Int. Ed., 2011, 50, 6851.

12 (a) Y. C. Shiang, C. C. Huang and H. T. Chang, Chem. Commun., 2009, 3437; (b) L. Jin, L. Shang, S. Guo, Y. Fang, D. Wen, L. Wang, J. Yin and S. Dong, Biosens. Bioelectron., 2011, 26, 1965; (c) Y. Li, Q. Ma, Z. Liu, X. Wang and X. Su, Anal. Chim. Acta, 2014, 840, 68; (d) X. Xia, Y. Long and J. Wang, Anal. Chim. Acta, 2013, 772, 81; (e) T. Wen, F. Qu,
N. B. Li and H. Q. Luo, Anal. Chim. Acta, 2012, 749, 56; (f) X. Wu, R. Li and Z. Li, RSC Adv., 2014, 4, 9935.

13 (a) Y. Sun and T. L. Huang, J. Polym. Res., 1995, 2, 47; (b) G. Cirillo, F. Puoci, M. Curcio, O. I. Parisi, F. Iemma, U. G. Spizzirri and N. Picci, Colloid Polym. Sci., 2010, 288, 689; (c) A. Waly, N. Y. Abou-Zeid, E. A. El-Alfy and A. Hebeish, Angew. Makromol. Chem., 1982, 103, 61.

14 (a) I. Díez, M. Pusa, S. Kulmala, H. Jiang, A. Walther, A. S. Goldmann, A. H. E. Müller, O. Ikkala and R. H. A. Ras, Angew. Chem., Int. Ed., 2009, 48, 2122; (b) J. Zhang, S. Xu and E. Kumacheva, Adv. Mater., 2005, 17, 2336.

15 (a) X. Wang, S. Xu and W. Xu, Nanoscale, 2011, 3, 4670; (b) C. Wang, Y. Wang, L. Xu, D. Zhang, M. Liu, X. Li, H. Sun, Q. Lin and B. Yang, Small, 2012, 8, 3137. 